Check for updates

Cite this: Soft Matter, 2018, 14,4453

Received 19th December 2017, Accepted 2nd March 2018

DOI: $10.1039 / \mathrm{c} 7 \mathrm{sm} 02488$

rsc.li/soft-matter-journal

\title{
Microfluidic solvent extraction of poly(vinyl alcohol) droplets: effect of polymer structure on particle and capsule formation $\dagger$
}

\author{
W. N. Sharratt, (D) ${ }^{a}$ A. Brooker, ${ }^{b}$ E. S. J. Robles $\mathbb{D}^{\mathrm{b}}$ and J. T. Cabral (D)*a
}

\begin{abstract}
We investigate the formation of poly(vinyl alcohol) microparticles by the selective extraction of aqueous polymer solution droplets, templated by microfluidics and subsequently immersed in a non-solvent bath. The role of polymer molecular mass (18-105 $\mathrm{kg} \mathrm{mol}^{-1}$ ), degree of hydrolysis (88-99\%) and thus solubility, and initial solution concentration $(0.01-10 \% \mathrm{w} / \mathrm{w})$ are quantified. Monodisperse droplets with radii ranging from 50 to $500 \mu \mathrm{m}$ were produced at a flow-focusing junction with carrier phase hexadecane and extracted into ethyl acetate. Solvent exchange and extraction result in droplet shrinkage, demixing, coarsening and phase-inversion, yielding polymer microparticles with well-defined dimensions and internal microstructure. Polymer concentration, varied from below the overlap concentration $c^{*}$ to above the concentrated crossover $c^{\star *}$, as estimated by viscosity measurements, was found to have the largest impact on the final particle size and extraction timescale, while polymer mass and hydrolysis played a secondary role. These results are consistent with the observation that the average polymer concentration upon solidification greatly exceeds $c^{\star *}$, and that the internal microparticle porosity is largely unchanged. However, reducing the initial polymer concentration to well below $c^{*}$ (approximately $100 \times$ ) and increasing droplet size yields thin-walled (100's of $\mathrm{nm}$ ) capsules which controllably crumple upon extraction. The symmetry of the process can be readily broken by imposing extraction conditions at an impermeable surface, yielding large, buckled, cavity morphologies. Based on these results, we establish robust design criteria for polymer capsules and particles, demonstrated here for poly(vinyl alcohol), with well-defined shape, dimensions and internal microstructure.
\end{abstract}

\section{Introduction}

Polymeric particles and capsules are versatile functional materials with applications ranging from cosmetics and personal care to coatings, ${ }^{1-4}$ photonics and drug delivery. ${ }^{5-8}$ Generally, these are formed by templating liquid droplets in emulsions or suspensions and subsequent polymerization of monomers, ${ }^{9}$ via a plethora of routes, or by physically or chemically inducing a liquid-solid transition in droplets of pre-formed polymer solutions, as for instance in spray drying. ${ }^{10}$ The rapid development of microfluidic techniques, ${ }^{11}$ has opened attractive routes for precise and reproducible emulsification, including multiple and hierarchical emulsions. $^{12,13}$ In turn, these have led to a multitude of novel approaches for particle formation: including by UV polymerisation

\footnotetext{
${ }^{a}$ Department of Chemical Engineering, Imperial College London, London SW7 2AZ, UK. E-mail: j.cabral@imperial.ac.uk

${ }^{b}$ Procter \& Gamble, Newcastle Innovation Centre, Newcastle-Upon-Tyne, NE 12 9TS, UK

$\dagger$ Electronic supplementary information (ESI) available. See DOI: 10.1039/ c7sm02488f
}

and lithography, ${ }^{14,15}$ interfacial polymerisation, ${ }^{16}$ complexation, ${ }^{17-19}$ gelation, ${ }^{16,20}$ and supramolecular assembly, ${ }^{21}$ detailed in a comprehensive book and numerous reviews. ${ }^{12,22-26}$ Amongst these, solvent extraction and phase inversion of polymer solutions within droplets provides a facile, versatile, and high-throughput approach for particle and capsule formation. In short, polymer solution droplets are initially suspended within an immiscible carrier phase, and subsequently extracted by immersion in a selective solvent. This extraction (or 'solvent displacement') removes solvent from the droplet, leading to a concentration of the polymer 'solute' and eventual particle or capsule formation.

Despite its simplicity, the process provides remarkable control of particle size and internal microstructure, but also shape and encapsulation and release properties. This control is, however, predicated on the thermodynamics of the ternary (or quaternary) system, comprising the polymer, solvent, extraction solvent (and carrier), and a range of transport and demixing processes.

Solvent extraction and phase inversion are extensively employed in the manufacture of polymeric membrane sheets, ${ }^{27,28}$ and the process shares many features with droplet extraction, in terms of demixing, coarsening and directional solidification in a ternary 
(or higher) system comprising a polymer, solvent and non-solvent. ${ }^{29}$ Droplet extraction is also reminiscent of the rapid precipitation of polymer solutions by 'flash nanoprecipitation' whereby opposing jets of a dilute polymer solution and non-solvent impinge at high Reynolds number, yielding rapid (turbulent) mixing and precipitation of nanoparticles (typically 10s-100s nm) of polymer, copolymers and composites, ${ }^{30-32}$ within ms timescales. By contrast, templating polymer solutions in droplets prior to extraction results in typical solidification timescales of seconds to minutes (and even hours), depending primarily on the partial miscibility of solvent and non-solvent system, and initial droplet size and concentration. Typical particle sizes are $10 \mathrm{~s}$ to $100 \mathrm{~s}$ of $\mu \mathrm{m}$ and exhibit a smooth polymer-rich skin. Their internal microstructure is largely controlled by the Péclet number, describing the relative timescales of shrinkage of the droplet interface and diffusion of solute(s) within, as well as internal convection and recirculation, and demixing and coarsening processes. The latter are triggered by the ingress of non-solvent and concentration of solute during droplet shrinkage. External flow has been shown to spatially modulate the rate of solvent extraction process causing droplets to depart from sphericity and form a range of anisotropic, dimpled and toroidal structures. ${ }^{33,34}$ Droplet extraction provides thus a powerful and versatile platform for the precise fabrication of polymeric particles and capsules.

We have previously examined the role of droplet size, polymer solution concentration and non-solvent extraction medium, during the investigation of the formation of porous polyelectrolyte microparticles, ${ }^{35,36}$ and proposed a justification for the resulting internal microstructure by a demixing pathway. Scaling relationships between the initial droplet size and polymer concentration and the resulting extraction timescales, particle dimensions and internal porosity were obtained, and a descriptive model introduced. Tuning the relative timescales for kinetic arrest and internal demixing, with concentration and non-solvent quality, was shown to provide effective control over the particle internal porosity. Recently, we have reported the formation polymer-colloid capsules with a variety of morphologies, controlled primarily by the respective component concentrations. We demonstrated that when dissolved, capsules with bicontinuous structures, accessed via a spinodal pathway, afforded a pulsed release profile of colloidal clusters over tuneable timescales.

The purpose of current paper is to investigate, for the first time, the role of polymer molecular mass $\left(M_{\mathrm{w}}\right)$ and solubility, given by the degree of hydrolysis, as well as droplet solution viscosity in the particle and capsule formation process. We select poly(vinyl alcohol) (PVA) as a model system for this study, since it is a water-soluble, biodegradable, ${ }^{37}$ neutral polymer, and extensively used as a barrier, coating and packaging material. Three PVA $M_{\mathrm{w}}$ and two degrees of hydrolysis are examined. Their overlap $c^{*}$ and concentrated crossovers $c^{* *}$ are determined from viscometry to define a range of representative polymer concentrations and viscosities of interest. We map the dependence of extraction timescales and resulting particle dimensions on polymer structure, initial concentration and droplet size.

\section{Materials and methods}

\section{Materials}

Hexadecane (ReagentPlus, $\geq 99 \%$ ), toluene (anhydrous, 99.8\%), octadecyltrichlorosilane ( $\geq 90 \%$ ), ethyl acetate (anhydrous, 99.8\%), and sorbitan mono-oleate ( $\operatorname{Span}{ }^{\mathrm{R}} 80$ ) were obtained from Merck. Acetone, ethanol, and isopropyl alcohol (all AnalaR, Normapur ${ }^{\circledR}$ ) were obtained from VWR International and NOA 81 (thiolenebased photoresist) was purchased from Norland Products, USA. All reagents were used as received. Poly(vinyl alcohol) (PVA) with average $M_{\mathrm{W}}=18,40$ and $105 \mathrm{~kg} \mathrm{~mol}^{-1}$ and degree of hydrolysis = 87-89 and 99\% was used as received (Sigma Aldrich no. 363170, 348406, 363073, 363138, 363081 and 363146). Deionized water was obtained from a Centra ELGA filtration system.

\section{Sample preparation}

Aqueous PVA solutions were prepared at $80{ }^{\circ} \mathrm{C}$ and $95{ }^{\circ} \mathrm{C}$ for, respectively $88 \%$ and $99 \%$ degree of hydrolysis, until full dissolution (up to $24 \mathrm{~h}$, for the highest $M_{\mathrm{w}}$ and degree of hydrolysis) under constant agitation. Water evaporation during dissolution was measured and accounted for in the final solution concentration. All samples were used within $24 \mathrm{~h}$, to minimise well-known ageing phenomena. ${ }^{38-41}$

\section{Viscosity}

Polymer solution viscosity was measured with a Brookfield DV-I prime viscometer, fitted with LV spindles and UL adapter, in a Couette geometry, at $25.0 \pm 0.5{ }^{\circ} \mathrm{C}$. Readings were taken after equilibration for 3 minutes and at spindle speeds between 0.3 and $60 \mathrm{RPM}$ - corresponding to shear rates of 0.2 to $73.4 \mathrm{~s}^{-1}$. Except for high concentration samples $(>15 \% \mathrm{w} / \mathrm{w})$, all solutions exhibited Newtonian behaviour. Specific viscosities were calculated as $\eta_{\text {sp }}=\eta_{\text {solution }} / \eta_{\mathrm{H}_{2} \mathrm{O}}-1$. The overlap concentration $\left(c^{*}\right)^{42}$ was estimated empirically by $\eta_{\mathrm{sp}}\left(c^{*}\right)=1$. This has been shown to yield values commensurate with values determined experimentally by dilute solution specific viscosities measurements fitted with the Huggins relation. ${ }^{43,44}$ The transition from the semi-dilute regime, characterised by the viscosity scaling law $\eta_{\mathrm{sp}} \propto c^{1.3}$, to the concentrated regime, with $\eta_{\mathrm{sp}} \propto c^{3.9-4.2}$, was determined by intersection of fits to the data with these scaling laws. Uncertainties in $c^{*}$ and $c^{* *}$ were estimated by concentrations compatible with maximum and minimum power law fits to the data within the relevant concentration regime (retaining a coefficient of variation $R^{2}>0.9$ ).

\section{Microfluidics}

Microfluidic devices were fabricated by thiol-ene closed-face photopolymerization as previously described, ${ }^{45}$ placing optical adhesive NOA81 between two glass slides followed by UV-A exposure. ${ }^{46}$ The channels were treated with a solution $(10 \% \mathrm{v} / \mathrm{v})$ of OTS in toluene and rinsed with toluene, isopropanol, ethanol and air dried before use. A flow-focussing geometry was used for emulsion droplet generation with microchannel dimensions: $400 \mu \mathrm{m}$ height, $450 \mu \mathrm{m}$ outlet channel width and a constriction width of $200 \mu \mathrm{m}$. The fluids were injected via syringe pumps (Harvard Apparatus PHD2000) with flow rates ranging from 
1-100 $\mu \mathrm{L} \min ^{-1}$. Water in oil (W/O) droplets and plugs were generated with $100-500 \mu \mathrm{m}$ radii by controlling dispersed and continuous phase flow rates. The dispersed phase consisted of an aqueous PVA solution $(\approx 0.01-10 \% \mathrm{w} / \mathrm{w})$ whilst the continuous phase comprised non-ionic surfactant $\operatorname{Span}{ }^{\mathbb{R}} 80$ (up to $2.5 \% \mathrm{v} / \mathrm{v}$ ) in hexadecane, to prevent droplet coalescence at collection. Tubing attached to the outlet channel immersed the droplets produced into a large bath $(25 \mathrm{~mL})$ of non-solvent ethyl acetate.

\section{Imaging and characterisation}

The microdevice was mounted on a motorised XY stage (Prior Scientific) of an inverted microscope (Olympus IX71) fitted with a CCD camera (Allied Vision, Manta G-235). Images were acquired at frame rates between 0.02 and $45 \mathrm{~s}^{-1}$, calibrated with a stage micrometer (Olympus, $0.01 \mathrm{~mm}$ OBX) and analysed using open source software (ImageJ). Selected particles and capsules were also imaged by Scanning Electron Microscopy (SEM) using a JEOL (JSM 6010LA) and Zeiss (Gemini Sigma300) microscopes in secondary electron imaging mode. Particles were dried for $24 \mathrm{~h}$, both intact and sectioned, and coated with a $10 \mathrm{~nm}$ chromium layer prior to imaging.

\section{Results and discussion}

\section{Microfluidic droplet generation and ex situ extraction}

A schematic of the formation of aqueous PVA solution droplets within a hydrocarbon carrier phase, hexadecane (HD), is depicted in Fig. 1a. Ethyl acetate (EA) was used as the extraction solvent due to its partial miscibility with water, full miscibility with the carrier phase and its non-solvency for the polymer. Initial solution viscosities ranged from 1 to $1800 \mathrm{mPa} s$ and, at the higher viscosities (>100 $\mathrm{mPa} \mathrm{s}$ ), droplet generation was found to depart from the 'squeezing' regime, ${ }^{47}$ which resulted in higher dispersity. For the droplet sizes and polymer concentrations investigated, the timescales of extraction were significantly longer (up to several $\mathrm{min}$ ) than typical residence times in our linear microchannels $(\leq 5 \mathrm{~s})$ at these flow rates and we thus opted to carry out droplet extraction ex situ, by immersion into a large non-solvent bath. The volume of the bath exceeded the total droplet volume by approximately 1000 times, thus preventing non-solvent saturation with water, and in turn inadvertent deviations of mutual solvent diffusion at the interface affecting drop shrinkage. ${ }^{48}$

The particle formation pathway is illustrated in Fig. 1b. This mechanism is expected to be general to ternary (or quaternary) systems, comprising polymer, solvent and selective solvent (and carrier), which fit the following criteria: the 'solvent' and 'selective' (or extraction) solvent are partially miscible, whilst the polymer is soluble in the 'solvent' but not in the 'selective' solvent, and the selective solvent is completely miscible with the carrier. For the PVA/ $\mathrm{H}_{2} \mathrm{O} / \mathrm{EA} /(\mathrm{HD})$ system investigated here, once the aqueous PVA solution droplets are immersed into EA, the selective extraction solvent, the droplet shrinks and thus the polymer concentration increases. However, these solvents are partially miscible and whilst the flux of water exiting the

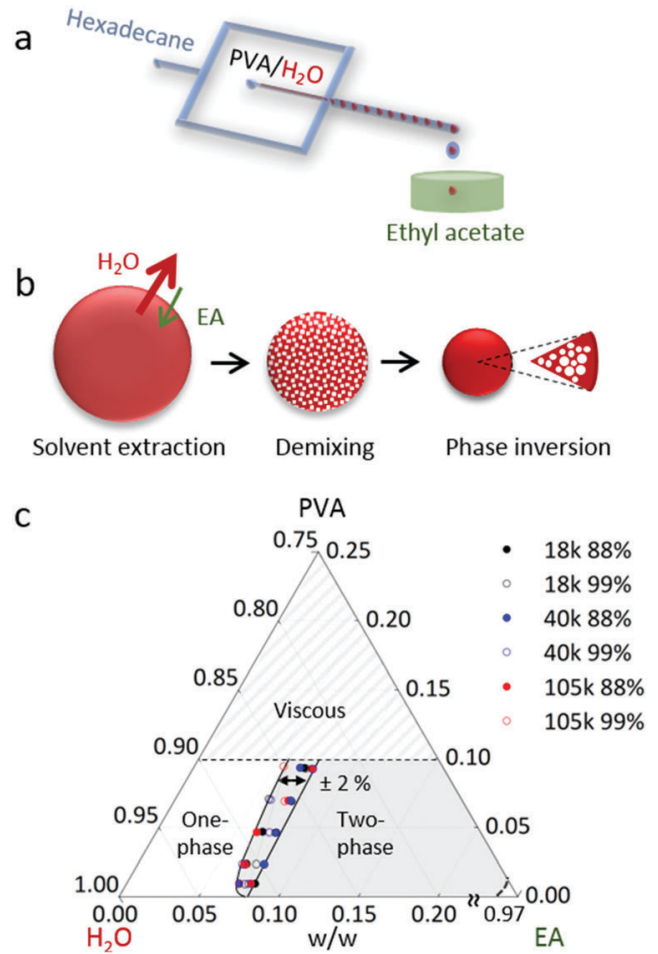

Fig. 1 (a) Schematic of the experimental setup depicting a flow-focussing microfluidic drop generator and an external precipitation bath. Aqueous polymer poly(vinyl alcohol) (PVA) solution droplets are suspended in a in carrier phase (HD) and extracted in non-solvent (EA). EA is miscible with $\mathrm{HD}$, partially miscible with $\mathrm{H}_{2} \mathrm{O}$ and a non-solvent for the polymer. (b) Schematic of solvent exchange at the liquid interface, demixing during droplet shrinkage and resulting particle structure. The resulting PVA particles typically consist of a smooth polymer-rich skin and an internally microporous structure. (c) Ternary phase diagram for PVA, $\mathrm{H}_{2} \mathrm{O}$ and EA measured by turbidity for six PVA samples of varying $M_{w}$ and degree of hydrolysis. The location of the phase boundary was within $\pm 2 \%$ in solvent composition for all PVA samples investigated.

droplet dominates, EA is also exchanged at the interface. This non-solvent ingress induces demixing and phase coarsening within the polymer solution droplet. The ternary phase behaviour of mixtures of PVA, $\mathrm{H}_{2} \mathrm{O}$ and EA is shown in Fig. 1c. As shown in the phase diagram, droplet shrinkage alone (along the $\mathrm{PVA}^{-} \mathrm{H}_{2} \mathrm{O}$ composition line) does not cross the two-phase boundary. The simultaneous extraction of solvent and phase separation results in a series of demixing steps, in a cascade along the stability line. Eventually, as only the solvent is removed from the droplet, the polymer-rich phase becomes majority and phase inversion takes place. This phase preferentially enriches the droplet interface leading to skin formation and kinetic arrest, upon further water removal. Droplets with appropriate polymer concentration (quantified below) thus yield polymer particles with a smooth surface and microporous internal structure. For the PVA samples investigated here, with $M_{\mathrm{w}}$ ranging from 18 to $105 \mathrm{~kg} \mathrm{~mol}{ }^{-1}$ and $88-99 \%$ hydrolysis, the phase boundary remains largely invariant, within $\pm 2 \% \mathrm{w} / \mathrm{w}$ of solvent composition for all samples measured, indicating that solvent/non-solvent interaction dominate phase stability. At room temperature, the solubility of EA in water is $\approx 9 \%$ and the solubility of water in EA is $\approx 3 \% \mathrm{w} / \mathrm{w}^{49}$ 
A representative time series of the droplet extraction process upon immersion into non-solvent, measured by optical microscopy, is shown in Fig. 2. The top row shows the gradual reduction in droplet radius over time, $R(t)$, and evolution of refractive index contrast until a polymer particle is formed, at extraction time $\tau$. The bottom row provides higher magnification images of the internal droplet structure during extraction, resolving the demixing and coarsening processes induced by the ingress of non-solvent. Nucleation and growth is the dominant demixing process for the conditions studied, evidenced by the gradual appearance of small droplets which undergo internal convection and coarsening within the large droplet, as extraction proceeds. While resolving the extraction pathway is complex, since it involves two phases within the droplet and the external medium, as well as spatio-temporally evolving concentration profiles, the droplet evidently undergoes a cascade of demixing and extraction steps, expected to take place along the phase boundary and thus nucleation should be the favoured mechanism. The characteristic size of a late-stage demixed structure in liquid droplets, estimated by microscopy, and that of a dried microparticle, observed by SEM (Fig. S1, ESI $\dagger$ ), agree well; yielding $\approx 3 \mu \mathrm{m}$ for a $\approx 200 \mu \mathrm{m}$ radius particle, shown here. Our observations with PVA solutions qualitatively agree with previous work on NaPSS and nanoparticle composites. ${ }^{50}$

\section{PVA solution viscosity}

In addition to solution thermodynamics, one might expect droplet extraction to depend on the polymer structure $\left(M_{\mathrm{w}}\right.$ and hydrolysis). We have therefore measured the dynamic viscosities for all six samples, as a function of concentration, and removed the contribution of solvent to the viscosity to afford specific viscosity values, as shown in Fig. 3a. The crossovers between dilute and semi-dilute, $c^{*}$, and then concentrated regimes, $c^{* *}$, were estimated by the changing power laws of the viscosity dependence on concentration, as discussed above. The behaviour is compatible with scaling predictions for neutral polymers in a good solvent. ${ }^{42}$ Evidently, increasing concentration and polymer $M_{\mathrm{w}}$ increases solution viscosity. Increasing degree of hydrolysis, from 88 and 99\%, also increases PVA solution viscosity, ${ }^{51}$ up to a factor of 2 , in particular for the higher $M_{\mathrm{w}}$ and above $c^{* *}$. For this narrow hydrolysis range, $c^{*}$ and $c^{* *}$ were unchanged, within measurement uncertainty, in agreement with

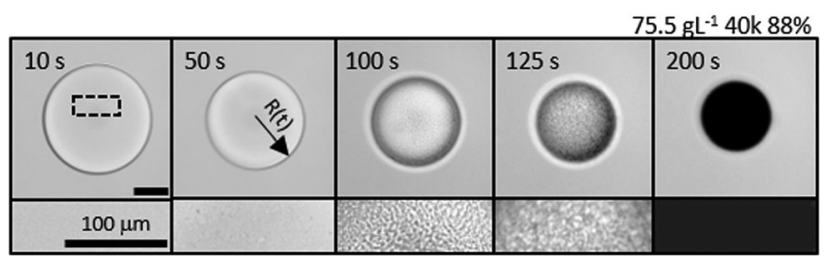

Fig. 2 (top row) Optical microscopy time series of the solvent extraction of a representative droplet of $40 \mathrm{k} 88 \%$ PVA solution $\left(75.5 \mathrm{~g} \mathrm{~L}^{-1}\right)$, showing droplet shrinkage, demixing, coarsening and kinetic arrest upon progressive densification. (bottom row) Corresponding higher magnification optical images of droplet morphology, in a region demarcated by the dashed rectangle.
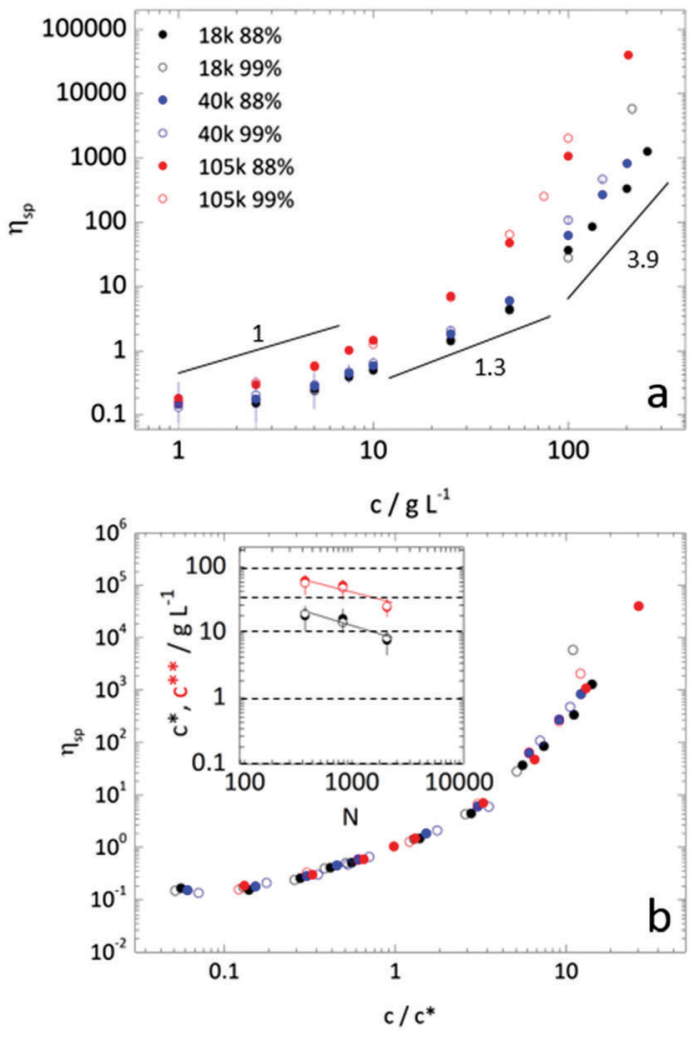

Fig. 3 (a) Dependence of specific viscosity of aqueous PVA solutions on polymer concentration for the $M_{w}$ and degree of hydrolysis investigated, including expected scaling exponents for neutral polymers in good solvent. Representative error bars are included for 40k 99\% PVA. (b) Specific viscosity dependence on scaled concentration $c / c^{*}$ for each PVA $M_{w}$ and degree of hydrolysis. The inset shows the dependence of $c^{\star}$ and $c^{\star \star}$ with degree of polymerization $(N)$ for $(\bullet) 88 \%$ and $(O)$ 99\% hydrolysis. Solid lines correspond to theoretical predictions for neutral polymers in good solvent, where $C^{*}, C^{* *} \sim N^{-0.76}$, and dashed horizontal lines indicate selected polymer solutions concentrations for droplet extraction experiments.

previous work..$^{52,53}$ Likely due to sample polydispersity, $c^{* *}$ was found to be approximately 3-4 greater than $c^{*}$, below the factor $\approx 10$ expected. ${ }^{44}$ Specific viscosity data are plotted by rescaled concentration $\left(c / c^{*}\right)$ in a master curve in Fig. $3 \mathrm{~b}$, and the dependence of $c^{*}$ and $c^{* *}$ with degree of polymerization $N$ are shown in inset. Representative polymer solution concentrations, indicated by the horizontal dashed lines at 100, 40, 1, 0.1 and $0.01 \mathrm{~g} \mathrm{~L}^{-1}$, were then selected for droplet extraction to examine the possible impact of solution regimes and relation to $c^{*}$ and $c^{* *}$ in particle or capsule formation.

\section{Parameterisation of the extraction process}

Fully modelling the droplet radius-time $R(t)$ relation is a complex moving boundary reaction-diffusion problem. ${ }^{54,55}$ While the classical Epstein-Plesset theory of droplet dissolution assumes Fickian diffusion and expects $R \sim t^{1 / 2},{ }^{56}$ and the HixsonCromwell model for solid particle dissolution yields linear kinetics, ${ }^{57}$ larger powers can also occur (in superdiffusion processes). In droplet extraction, in addition to dissolution, internal convection, phase separation and inversion, interfacial tension and polymer enrichment at the surface are all expected 
to play a role in the shrinkage profile. We therefore employ a minimal descriptive model, introduced previously, ${ }^{35,36,50}$ to parameterise droplet shrinkage and particle formation:

$$
R(t)=\left(R_{0}-R_{\infty}\right)\left(1-\frac{t}{\tau}\right)^{\alpha}+R_{\infty}
$$

where $R_{0}$ is the initial droplet radius, $R_{\infty}$ the final particle size, $\tau$ the extraction time (defined as the time droplet shrinkage ceases, i.e. $\mathrm{d} R / \mathrm{d} t=0$ ), and $\alpha$ is a hyperbolicity parameter, which empirically accounts for non-Fickian diffusion associated with dynamic interfacial effects, hydrodynamics, demixing and coarsening, and inhibited transport discussed above. Previous studies on microdroplet dissolution in partially miscible solvents, extending the Epstein-Plesset theory to a range of liquid-liquid systems, ${ }^{48,58,59}$ validated the assumption that the moving droplet interface, and transport term in the diffusion equation, can be neglected for cases where solvent diffusion is rate limiting. This applies to cases where the solvent concentration profile extends to large distances $(>R)$ and diffusion is much faster than droplet dissolution timescales (for pure liquid droplets). In our experiments, the presence of polymer 'solute' further hinders diffusion and thus extraction kinetics. Fig. 4a shows representative extraction data, $R(t)$, for $40 \mathrm{~g} \mathrm{~L}^{-1}, 40 \mathrm{k} 88 \% \mathrm{PVA}$, droplets of various $R_{0}$. The lines are fits to eqn (1). Fig. $4 \mathrm{~b}$ replots these data scaled according to the model yielding a single master curve, for all $R_{0}$.

Inspection of final particle sizes and available polymer mass (of known density) ${ }^{60}$ in the initial solution indicates that the resulting particles are not compact and must contain void
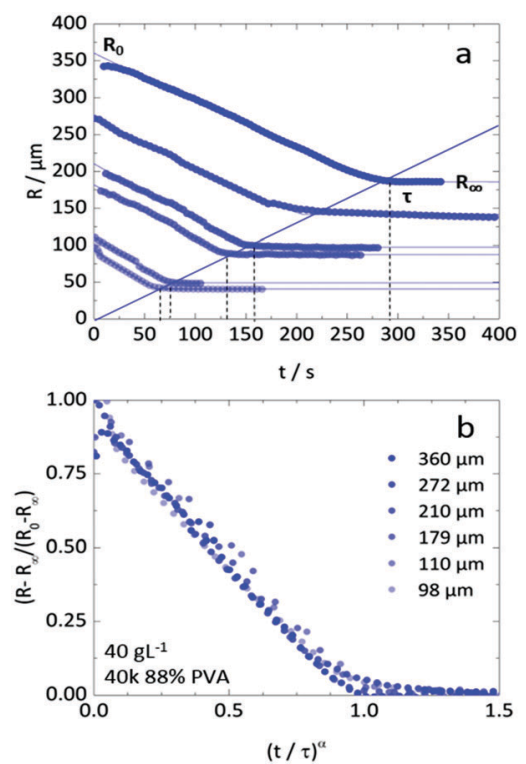

Fig. 4 (a) Droplet radius plotted as a function of extraction time for representative $40 \mathrm{~g} \mathrm{~L}^{-1}$ PVA (40k $88 \%$ ) solution droplets of various initial size. A descriptive model (eqn (1)) was fitted to the data to parametrise the extraction process. The initial droplet radius $\left(R_{0}\right)$, final particle radius $\left(R_{\infty}\right)$ and extraction time $(\tau)$ are indicated for the largest droplet. The solid line indicates a linear dependence of $\tau$ on initial droplet size given by $\tau \approx$ $(0.77 \pm 0.01) R_{0}$. (b) Rescaled data plotted $\left(\frac{R_{t}-R_{\infty}}{R_{0}-R_{\infty}}\right)$ against $\left(\frac{t}{\tau}\right)^{\alpha}$, as expected from eqn (1).
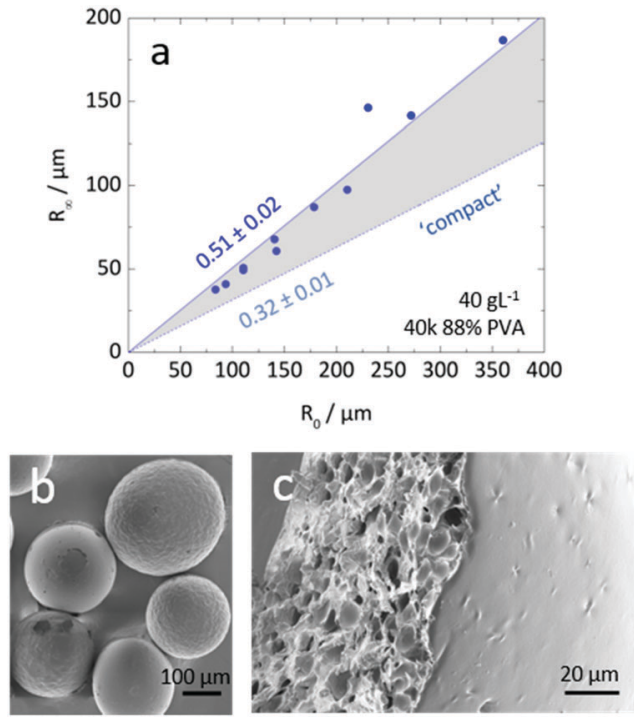

Fig. 5 (a) Dependence of $R_{\infty}$ on $R_{0}$ for a $40 \mathrm{~g} \mathrm{~L}^{-1} 40 \mathrm{k} 88 \%$ PVA solution, and expectation for the radius of equivalent 'compact' particles calculated by mass conservation and the known density of PVA. The gap between the two linear fits provides an estimate of average porosity. (b) SEM image of representative PVA particles. (c) SEM micrograph of a sectioned 40k $88 \%$ PVA particle confirmed the prediction of void volume; the particle consisted of a smooth polymer-rich skin and a porous internal structure with $\approx \mu$ m sized voids

volume. Fig. 5a presents the dependence of the final particle radius $R_{\infty}$ on initial droplet radius $R_{0}$ for $40 \mathrm{k} 88 \%$ PVA solutions. The expectation of particle size based on mass is also given, indicating that particles contain approximately $1-(0.3 / 0.5)^{3} \approx$ $75 \%$ void volume. Fig. $5 \mathrm{~b}$ shows the relatively smooth external polymer layer of the particles, while Fig. $5 \mathrm{c}$ shows an image of a dried cross-sectioned particle, exhibiting considerable internal porosity. As depicted in Fig. 1b, this internal microstructure arises due to the ingress of non-solvent and consequent demixing, coarsening and kinetic arrest of the phase-separated structure. Under the conditions employed, the particles exhibit a smooth polymer skin, which is compatible with an effective Péclet number for the extraction process, $\left(\mathrm{Pe}=\frac{\left(R_{0}-R_{\infty}\right)^{2}}{D \tau}\right) \gg 1$, stemming from the accumulation of polymer at the receding front (where the polymer diffusion coefficient is approximated by Stokes-Einstein in water and up to $c^{*}$ ). While the formation of a skin is expected from the rapid extraction process, the thickness of the skin layer is expected to be set by the overall polymer concentration, $R_{0}$, and the coarsening timescale. Overall, we find that the structure of the resulting particles is spatially heterogeneous, with a smooth polymer skin and a microporous internal structure. SEM imaging suggests that for initial PVA concentrations above $\geq 0.1 \mathrm{~g} \mathrm{~L}^{-1}$, the overall particle shape remains spherical, and the microstructure is largely uniform, likely due to convection before kinetic arrest.

\section{Initial droplet concentration (and $\boldsymbol{R}_{\mathbf{0}}$ ) determines $\boldsymbol{R}_{\infty}$}

Fig. 6a compares the extraction of droplets of similar initial size $\left(R_{0} \approx 200 \mu \mathrm{m}\right)$ and PVA concentration varying from 10 to 
$100 \mathrm{~g} \mathrm{~L}^{-1}$, corresponding to approximately 1 to $10 \%$ polymer by mass. The extraction profiles $R(t)$ are shown in Fig. 6b, establishing that droplets of lower polymer concentration take longer time and yield smaller particles, as could be expected. Within this concentration range, no surface buckling is observed, and the particles are largely spherical. The dependence of $R_{\infty}$ on $R_{0}$ is shown in Fig. 6c and which is found to be linear, with a concentration dependent slope, following from the observations above. The hyperbolicity parameter $\alpha$ is shown in Fig. 6d, accounting for the curvature of the extraction profile, and ranged from 0.64 to 1.4 respectively for pure water and the highest PVA concentration. Finally, Fig. 6e establishes the extraction timescale, defined as shown in Fig. 4a, for the three PVA concentrations. The relative scatter in the data is thought to arise from the presence of the carrier phase and droplet crowding, which may modulate the (ternary) solvent concentration profile surrounding the droplets. Nevertheless, the $\tau\left(R_{0}\right)$ dependence is found to be linear in all cases, as previously found for NaPSS, ${ }^{35,36}$ and the data for initial polymer $c>c^{*}$ is found to overlap within measurement uncertainty. Droplets with initial PVA concentration of $10 \mathrm{~g} \mathrm{~L}^{-1}\left(<c^{*}\right)$ take comparatively 3-4 times longer to form particles.

We note that the extraction timescales $\tau$ presented in the main paper were obtained under quiescent conditions, i.e. for stationary droplets in the absence of external flow, and are thus solely diffusive. Additional stirring of the extraction medium can trivially expedite the removal of the solvent from the vicinity of the droplet, adding a convective term, and thus result in shorter $\tau$ (as detailed in Fig. S2, ESI $\dagger$ ).
One might have expected longer $\tau$ to enable further coarsening within the demixed droplet. However, cross-sectional SEM measurements revealed that, within the concentration range $10-100 \mathrm{~g} \mathrm{~L}^{-1}$, PVA particles exhibited similar internal porosity, as shown in Fig. S3 (ESI $\dagger$ ). We interpret this observation as a result of the kinetic arrest taking place at (average) polymer concentrations which are consistently above $c^{* *}$. Similar conclusions have been drawn for observed the porosity of non-solvent induced precipitation of poly(styrene) particles. ${ }^{61}$ At sufficiently low initial concentrations, however, this trend no longer applies, and only a thin polymer skin is formed, as discussed below. Overall, these results combined enable the predictive design of microporous PVA particles with controlled dimensions $\left(R_{\infty}\right)$ and timescales $(\tau)$, across various concentrations of a given $M_{\mathrm{w}}$ polymer.

\section{Limited effect of $\boldsymbol{M}_{\mathrm{w}}$ and degree of hydrolysis}

Fig. 7a extends the analysis of the dependence of $R_{\infty}$ on $R_{0}$ to examine the effect of $M_{\mathrm{w}}$ and degree of hydrolysis at two representative concentrations ( 40 and $100 \mathrm{~g} \mathrm{~L}^{-1}$ ). For the range investigated, we find that the particle formation process does not depend on chain length and hydrolysis, within measurement uncertainty. Instead, initial droplet concentration is found to define the proportionality between $R_{\infty}$ and $R_{0}$. We attribute this insensitivity to the fact that solidification (as defined by $\tau$ ) is reached at droplet concentrations well above $c^{* *}$ and that, therefore, overall polymer dimensions become unimportant. Insensitivity to the degree of hydrolysis is potentially attractive, as polymer dissolution depends strongly on it and may provide facile access to tunable release properties. Detailed calculations
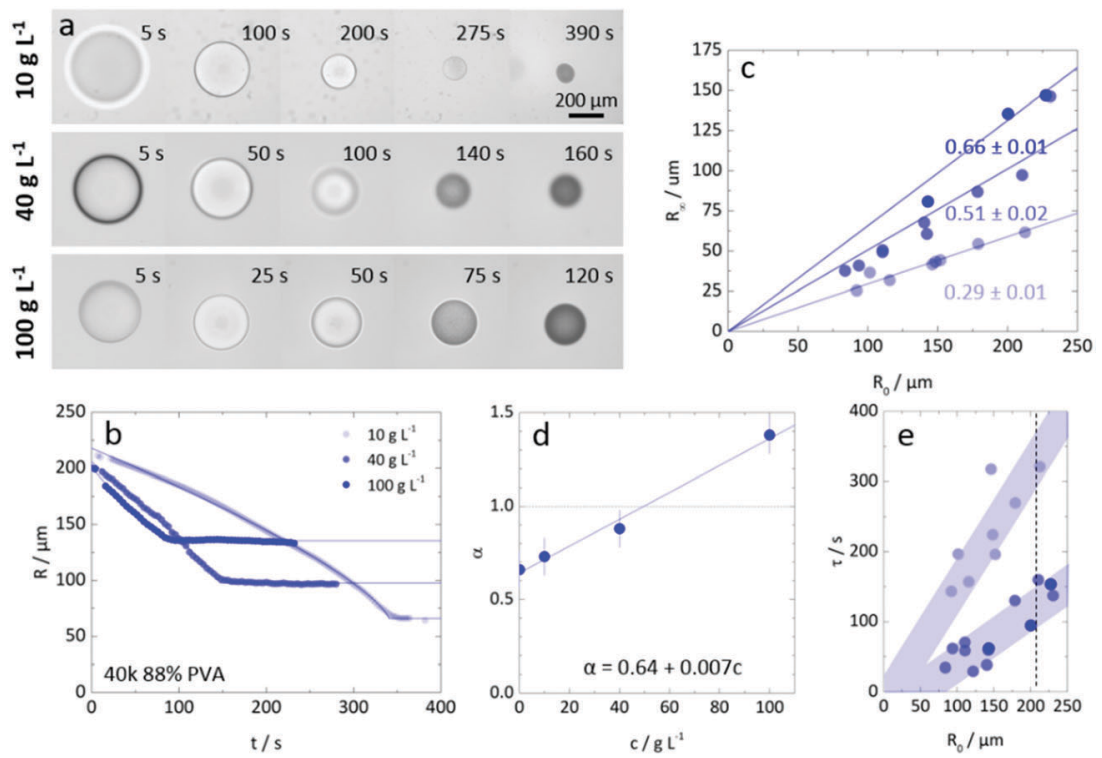

Fig. 6 (a) Optical microscopy images of 40k 88\% PVA solution extractions for droplets of 10,40 and $100 \mathrm{~g} \mathrm{~L}^{-1}$ concentrations and initial size $R_{0} \approx$ $200 \mu \mathrm{m}$. (b) Radius-time plots, with descriptive model fits (solid lines) for the three representative droplets shown in (a). (c) Variation in $R_{\infty}$ with $R_{0}$ for the three polymer concentrations investigated. Linear fits (solid lines) were plotted with an imposed condition that at $R_{0}=0, R_{\infty}=0$. The apparent linear variation in $R_{\infty}$ with $R_{0}$ depended on the polymer concentration. (d) Variation of $\alpha$ with concentration for the representative extractions and at zero polymer concentration (pure water). Within experimental error, $\alpha$ increased linearly with polymer concentration. (e) Dependence of $\tau$ on $R_{0}$ for the three concentrations investigated. In the absence of theory to guide expectation of the variation of timescale with $R_{0}$, shaded areas are used to guide the eye and illustrate the linear trends. With scatter in the data, concentrations below $c^{*}$ resulted in higher extraction timescales whilst, above $c^{\star}$ the timescales were broadly the same. 

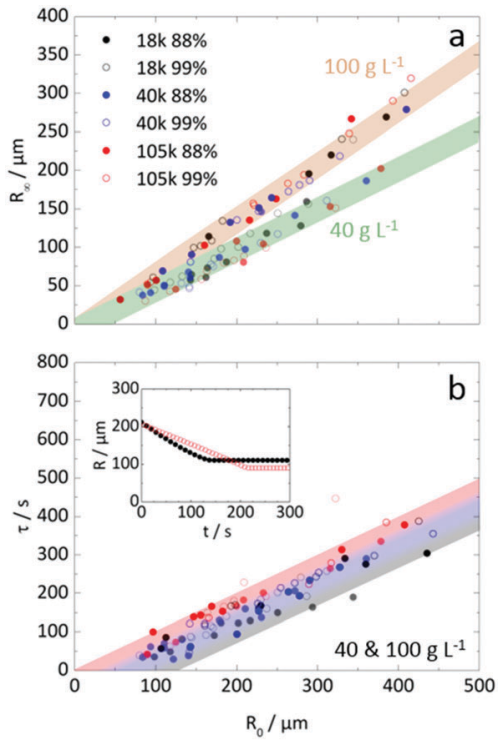

Fig. 7 (a) Comparison of final microparticle radius to initial droplet size for $40 \mathrm{~g} \mathrm{~L}^{-1}$ and $100 \mathrm{~g} \mathrm{~L}^{-1}$ solution droplets. Bands were superimposed on the data to indicate the linearity of $R_{\infty}$ with increasing $R_{0}$ and to illustrate the minor differences, within experimental uncertainty, as a function of molecular weight and degree of hydrolysis. For $40 \mathrm{~g} \mathrm{~L}^{-1}$ solutions the microparticles were approximately $50 \%$ of the size of the initial droplet whilst for $100 \mathrm{~g} \mathrm{~L}^{-1}$ the microparticles were approximately $70 \%$ of $R_{0}$. (b) Extraction timescale $(\tau)$ as a function of $R_{0}$ for 40 and $100 \mathrm{~g} \mathrm{~L}^{-1}$ solution droplets. As in (a) $40 \mathrm{~g} \mathrm{~L}^{-1}$ data points are indicated by the lighter colour. Trivially, extraction time increased with droplet size. Whilst no discernible difference in $\tau$ could be observed between 40 and $100 \mathrm{~g} \mathrm{~L}^{-1}$ droplets and with degree of hydrolysis, $\tau$ increased with increasing $M_{w}$. This is highlighted by the shaded band superimposed on the data. The higher $M_{w}$ droplets generally extracted more slowly, presumably due to the reduced diffusivity of solvent molecules through the much higher viscosity solution. (inset) Comparison of $40 \mathrm{~g} \mathrm{~L}^{-1}$ droplet radii for similar sized ( $\left.205 \mu \mathrm{m}\right) 18 \mathrm{k}$ $88 \%$ (black circles) and 105k 99\% (open red circles) during the extraction. Larger $\left|\frac{\mathrm{d} R}{\mathrm{~d} t}\right|$ for the lower molecular weight $(t<\tau)$, and viscosity, droplet resulted in a shorter extraction time and slightly larger final particle radius.

of the evolution of (average) polymer concentration from initial droplet to final particle are shown in Fig. S4a (ESI†). For both initial concentrations examined, all PVA solutions were above $c^{*}$ at $40 \mathrm{~g} \mathrm{~L}^{-1}$ and all were above $c^{* *}$ at $100 \mathrm{~g} \mathrm{~L}^{-1}$, as shown in inset of Fig. $4 \mathrm{~b}$. We do not observe a common (average) polymer concentration at solidification, nor a single criterion for the (average) viscosity upon particle formation (Fig. S4b, ESI $\dagger$ ). Instead a range of concentrations above $c^{* *}$ are found, as expected since the particles are internally heterogeneous, due to demixing and skin formation.

The $\tau\left(R_{0}\right)$ dependence on $M_{\mathrm{w}}$ is shown in Fig. $7 \mathrm{~b}$. In first approximation, given the (intrinsic) scatter in the data, extraction times appear to depend only on $R_{0}$ and not on $M_{\mathrm{w}}$, degree of hydrolysis nor concentration (for this range). Closer inspection of the data reveals, on average, that increasing $M_{\mathrm{w}}$ correlates with marginally longer $\tau$ as illustrated by the gradient band encompassing the data. The inset of Fig. 7b, illustrates this variation of $\tau$ for two droplets of the same initial size and concentration, for which the lower $M_{\mathrm{w}}$ droplet forms a particle earlier. The scatter in the data likely originates from some (minor) variability of the diffusion profile around each droplet, and can be affected by crowding, proximity to surfaces, and remnant carrier phase. Decreasing concentrations $\leq 10 \mathrm{~g} \mathrm{~L}^{-1}$ results in significant increases in $\tau$ at constant $R_{0}$, (Fig. S5, ESI $\dagger$ ), so this approximate insensitivity holds for $c>c^{*}$. At lower concentrations, solvent exchange occurs for longer times before the droplet concentration reaches the phase boundary and demixes, forming a polymer-rich phase. A subsequent section examines these conditions in more detail.

\section{Does concentration or viscosity fix the timescale and size?}

Fig. 8 examines the possibility that droplet solution viscosity alone controls the time scale of extraction, before solidification takes place. A fixed solution viscosity was selected (15 $\pm 1 \mathrm{mPa} \mathrm{s})$ and, from Fig. 3a, a suitable combination of $M_{\mathrm{w}}$ and polymer concentration (all above $c^{* *}$ ) to satisfy that requirement. The extraction of droplets of $R_{0} \approx 200 \mu \mathrm{m}$ droplets yields decreasing $R_{\infty}$ and increasing $\tau$ with increasing $M_{\mathrm{w}}$ (and thus reducing

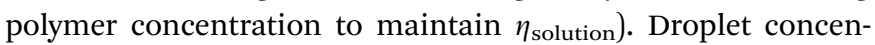
tration (and not initial viscosity) emerges as the main parameter to tune particle size $R_{\infty}$ and timescale $\tau$ (which are closely linked as shown in Fig. 6e and 7b). Despite the difference in $R(t)$ profiles, the final average concentration of polymer within the particles was found to reside within $\pm 5 \%$ of each other, despite the concentration varying by more than a factor of 2 between the lowest and highest $M_{\mathrm{w}}$ of PVA droplets. These results are consistent with our previous analysis of polymer content in the particles, where we found, within experimental uncertainty, no significant trends with $M_{\mathrm{w}}$ or degree of hydrolysis. Estimations of the viscosity within the droplet at the onset of solidification, shown in Fig. S4b (ESI †), provide further evidence for the concentration, not viscosity, controlling particle size.

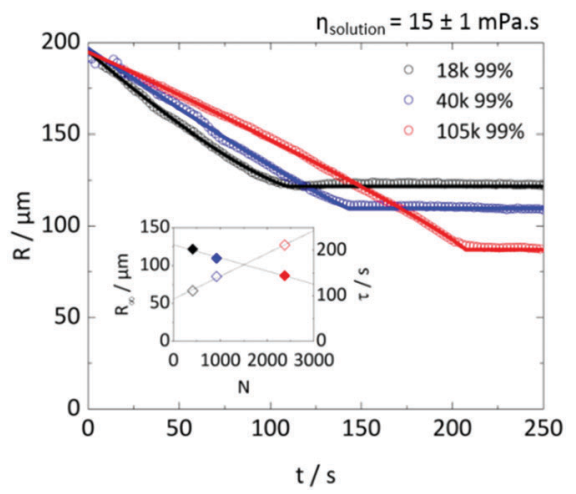

Fig. 8 Radius-time plots for three PVA solution droplets with varying $M_{\mathrm{w}}$ (and $R_{0} \approx 200 \mu \mathrm{m}$ and $99 \%$ degree of hydrolysis). The solution viscosities were matched to $15 \pm 1 \mathrm{mPa}$ s (or $\eta_{\mathrm{sp}}=16 \pm 1$ ) and therefore polymer concentration varied across the droplets: $98 \mathrm{~g} \mathrm{~L}^{-1}$ for $18 \mathrm{k}, 69 \mathrm{~g} \mathrm{~L}^{-1}$ for $40 \mathrm{k}$, and $34 \mathrm{~g} \mathrm{~L}^{-1}$ for $105 \mathrm{k}$. Inset shows $R_{0}$ (open diamonds) and $\tau$ (closed diamonds) as a function of degree of polymerisation $N$, in colours following the main figure for $M_{w}$. Lines are guides to the eye. Droplets solutions with higher $M_{w}$, at the same viscosity (and therefore lower polymer concentration), form smaller particles and extract over longer times. 


\section{Anisotropic extraction and particle deformation}

In the data presented so far, droplet crowding, hindered diffusion, solvent exchange near walls or surfaces, and partial wetting of the substrate, were carefully avoided (or data known to be affected were excluded from analysis). We emphasise, however, that most droplet extraction experiments will be otherwise inadvertently affected by these factors. For instance, upright optical microscopy will generally suggest the formation of a spherical particle, and a side view of the droplet is needed to resolve its 3-dimensional shape and evolution during extraction, in particular on or near a surface. We next discuss these effects and their impact on droplet extraction and resulting particle shape. The main indicators of such non-ideal effects are (i) non-monotonic decreases in $R(t),{ }^{35}$ and (ii) poor repeatability of $\mathrm{d} R(t) / \mathrm{d} t$ between measurements (generally the steepest slopes, related to solvent mutual diffusion, are most reliable). Further to this, differences are most pronounced for higher $R_{0}(>300 \mu \mathrm{m})$ and experimentally found to coincide with droplet-to-particle deformation away from sphericity, yielding cavities and/or ellipsoids to accommodate volume reduction. These effects are illustrated in Fig. 9a for the extraction or a relatively large droplet partly wetting the substrate during extraction. The $R(t)$ profile exhibits several "kinks" (discontinuities in slope) which can be mapped onto contact line pinning and deformation events by optical microscopy. The formation of cavities and deformation of particles were consistently observed in instances where the substrate was inhomogeneously coated with OTS. Anisotropic solvent exchange and removal, hindered by the impermeable substrate, is the likely cause for cavity formation under the conditions examined. Under

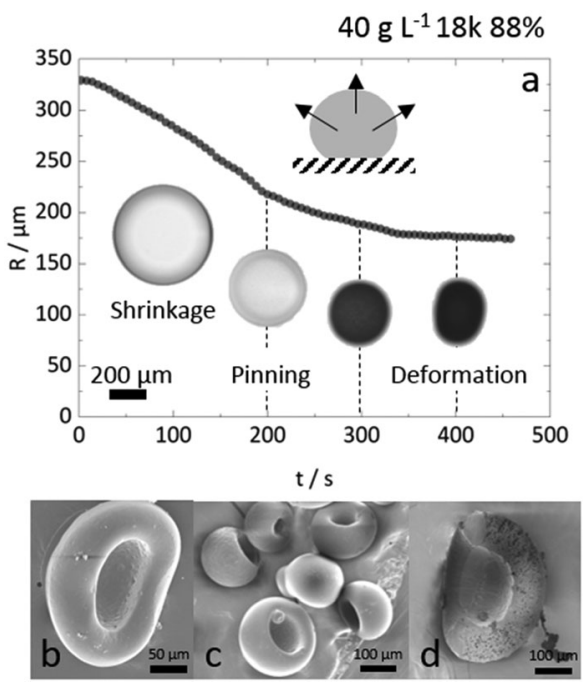

Fig. 9 (a) Non-monotonic extraction profile $R(t)$ of a partially wetting droplet ( $40 \mathrm{~g} \mathrm{~L}^{-1} 18 \mathrm{k} 88 \%$ hydrolysed PVA) with projected $R_{0} \approx 330 \mu \mathrm{m}$, on an inhomogeneous OTS-treated substrate. Optical images of the droplet are shown below. Cavity formation appears to arise due to anisotropic extraction. (b) SEM images of the substrate side of representative deformed particles with large cavity $\left(10 \mathrm{~g} \mathrm{~L}^{-1} 40 \mathrm{k} 88 \%\right)$. (c) Ensemble, with a range of particle sizes $\left(R_{\infty} \approx 160-360 \mu \mathrm{m}\right)$, for similar cap-like particles with voids (100 $\mathrm{g} \mathrm{L}^{-1} 40 \mathrm{k} 88 \%$ ). (d) Sectioned particles (as shown in panel c) were found to retain the porous internal microstructure characteristic of this concentration range. conditions of constant contact diameter drying, ${ }^{62,63}$ and polymer deposition and adhesion on the substrate, pinning of the threephase line leads to simultaneously contact angle and volume reduction. Representative particles are shown in Fig. 9b and c. The projected area observed by microscopy is thus no longer representative of a shrinking spherical droplet and particle. Further, hindered diffusion near the surface delays skin formation, breaks symmetry and thus facilitates a buckling transition at the boundary. ${ }^{64,65}$ Similar effects have been previously observed in the gastrulation of sea-urchin embryos, ${ }^{66}$ a model system for morphogenesis, but also in the formation of polymer particles by solvent extraction under flow, ${ }^{31}$ or in evaporation from a supramolecularly cross-linked polymer-skin. ${ }^{33,67,68}$ Asymmetry in the removal of solvent, arising from stagnation points upon flow or stratification at the air-liquid interface was found to yield buckled or, in the most extreme cases, toroidal structures.

In our measurements, droplet deformation and cavity formation were mitigated for smaller $R_{0}$ and higher $M_{\mathrm{w}}$, as shown in Fig. S6 (ESI $\dagger$ ). Fig. 9c shows that cavity formation did not disrupt the expected internal microstructure, suggesting that demixing and buckling or deformation are largely decoupled. A systematic examination of the role of sessile droplet size and polymer concentration on droplet shape, substrate wettability, and consequences for extraction kinetics is provided in Fig. S7-S9 (ESI $\dagger$ ).

These results show that, overall, the isotropy of solvent extraction can be trivially disturbed by the presence of an impermeable surface and, in addition to flow (with respect to the droplet), provides a facile way to create dimpled particles.

\section{Towards the limit of low polymer concentration}

We next consider the effect of decreasing the initial polymer concentration well below $c^{*}$, to examine the feasibility of capsule generation with ultrathin polymer membranes. Fig. 10 illustrates droplet extraction as initial polymer concentration is decreased by up to a factor of 1000 with respect to the data shown so far. At the lowest concentration $\left(0.1 \mathrm{~g} \mathrm{~L}^{-1}\right)$ and largest droplet radius (700 $\mu \mathrm{m})$ examined, the extraction time becomes extremely long

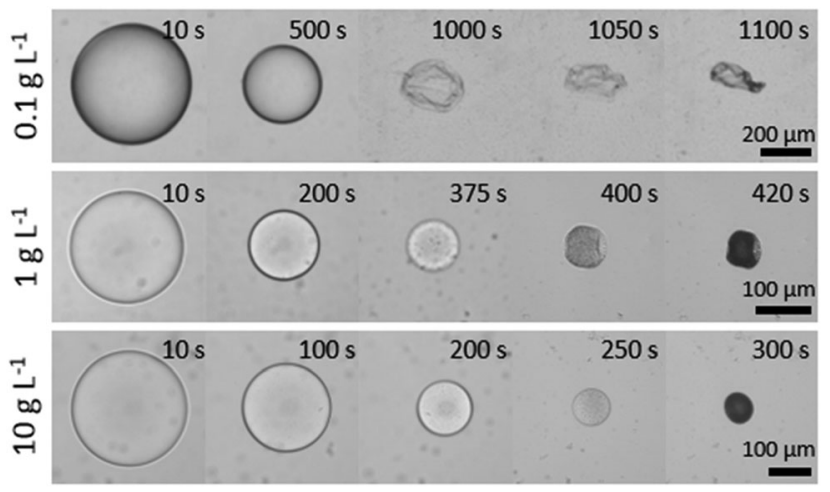

Fig. 10 Time-series of droplet extraction for concentrations below $C^{\star}$, for 40k 88\% PVA solutions (concentrations 10, 1 and $0.1 \mathrm{~g} \mathrm{~L}^{-1}$ correspond to $\approx 1,0.1$ and $0.01 \% \mathrm{w} / \mathrm{w}$ polymer). At the lowest concentration studied, a capsule with a thin $(\approx 300 \mathrm{~nm})$ polymer skin crumples at long extraction times. 
$(\approx 20 \mathrm{~min})$ and yields a polymer capsule with a thin $(\approx 300 \mathrm{~nm})$ polymer-rich skin (a "balloon"), which eventually collapses upon further extraction. The hyperbolicity parameter $\alpha$ is slightly larger than that for pure water, as expected for hindered diffusion. Upon increasing concentration by one order of magnitude, to $1 \mathrm{~g} \mathrm{~L}^{-1}$, the particle skin was evidently substantial enough to prevent crumpling, but nonetheless buckles to accommodate the final volume reduction. As per our previous data, at and above $10 \mathrm{~g} \mathrm{~L}^{-1}$ (approximately 1\% w/w) particles become predominately spherical and internally microporous.

\section{Conclusions}

The role of polymer architecture ( $M_{\mathrm{w}}$ and hydrolysis), concentration and droplet radius were examined in the formation of PVA microparticles by droplet extraction. Aqueous PVA solution droplets were formed in a planar flow-focussing device, with a carrier phase (hexadecane, HD), and extracted by immersion into a selective solvent (ethyl acetate, EA), which is partially miscible with $\mathrm{H}_{2} \mathrm{O}$, fully miscible with $\mathrm{HD}$ and immiscible with PVA. By mapping the phase behaviour of ternary mixtures of PVA/EA/ $\mathrm{H}_{2} \mathrm{O}$ and the viscosity of PVA solutions, as a function of polymer mass and backbone hydrolysis, the extraction pathway can be estimated. The particle formation mechanism can be rationalised in terms of a series of steps: (i) solvent exchange and droplet shrinkage, (ii) demixing caused by non-solvent ingress, (iii) internal coarsening and convection, (iv) interfacial enrichment of the polymer-rich phase, creating a smooth polymer skin, and eventual (v) phase inversion and kinetic arrest. Demixing and droplet shrinkage occur simultaneously, and the relative rate of these processes is thought to determine the internal porosity of the templated PVA microparticles. From viscosity measurements, $c^{*}$ and $c^{* *}$ for the PVA samples of various $M_{\mathrm{w}}$ and hydrolysis were determined. Representative concentrations below, within and above these values were selected to assess their possible impact into particle formation. We find that, for the range investigated, initial polymer solution concentration primarily determined the final particle size, for the same initial droplet size, while variations $M_{\mathrm{w}}$ and degree of hydrolysis had a minor effect. Initial concentrations below $c^{*}$ resulted in longer extraction times, while above $c^{*}$ or $c^{* *}$, small variations in extraction time were observed. For initial polymer concentrations above $c^{*}$, the average polymer concentrations at the onset of skin formation and in the final particle vastly exceed $c^{* *}$. We rationalise the relative insensitivity of particle formation to $M_{\mathrm{W}}$ by fact that in the concentrated regime, overall polymer dimensions become unimportant. We could rule out the initial viscosity affecting the solvent extraction process by matching initial viscosity above $c^{* *}$ and adjusting polymer concentration for droplets of differing $M_{\mathrm{w}}$. These results supported our hypothesis that concentration was the major determining factor in the extraction process.

In selected experiments, droplet radius was found to decrease non-monotonically during the extraction, and result in the formation of particles with a cavity and deformed shapes.

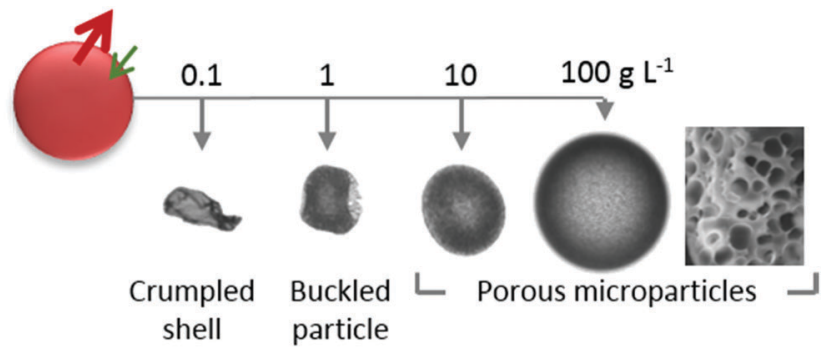

Fig. 11 Summary of the concentration effect on particle morphology. Well within the dilute regime, progressive dehydration of PVA solution droplets result in formation of thin polymer skins which subsequently collapse into crumpled structures. At higher concentrations, within the dilute regime, particles buckled during the latter stages of the extraction whilst, close to the overlap concentration, and in the semi-dilute and concentrated regimes, particles with increasingly circular cross-sections. Particles formed were internally porous with from the ingress of nonsolvent and demixing occurring during the extraction.

This effect is observed for anisotropic extractions near solventimpermeable surfaces and is exacerbated by the partial substrate wetting. Shape deformation and cavity formation take place to accommodate the particle volume reduction under pinning condition, and we found this effect to be most pronounced at low $M_{\mathrm{w}}$ and large $R_{0}$. Buckled and toroidal particles have also been reported to form under prescribed flow conditions, ${ }^{33,34,67,68}$ and collectively these methods provide a number of facile routes for the fabrication of anisotropic particles.

Reducing polymer concentration to well below $c^{*}$, led to an increase in extraction timescales and the formation of capsules with a thin $(\sim 100 \mathrm{~s} \mathrm{~nm})$ polymer-skin, and crumpled and buckled structures. As illustrated in Fig. 11, this work establishes robust design principles and scaling relations for the precise generation of PVA microparticle and capsules, with potential applications in coatings, consumer goods and agrichemicals.

\section{Conflicts of interest}

There are no conflicts to declare.

\section{Acknowledgements}

We thank the EPSRC and Procter and Gamble for financial support, and Carlos G. Lopez for discussions on rheology and scaling of polymer solutions. We also thank Dr Mahmoud Ardakani for training and use of the electron microscopy facility at Imperial College.

\section{Notes and references}

1 X. Fei, H. Zhao, B. Zhang, L. Cao, M. Yu, J. Zhou and L. Yu, Colloids Surf., A, 2015, 469, 300-306.

2 R. Tekin, N. Bac and H. Erdogmus, Macromol. Symp., 2013, 333, 35-40.

3 S. K. Ghosh, Functional Coatings, Wiley-VCH Verlag GmbH \& Co. KGaA, 2006, pp. 187-220, DOI: 10.1002/3527608478.ch6. 
4 I. van Driessche and S. Hoste, Functional Coatings, WileyVCH Verlag GmbH \& Co. KGaA, 2006, pp. 259-296, DOI: 10.1002/3527608478.ch8.

5 S. De Koker, R. Hoogenboom and B. G. De Geest, Chem. Soc. Rev., 2012, 41, 2867-2884.

6 A. Kumari, S. K. Yadav and S. C. Yadav, Colloids Surf., B, 2010, 75, 1-18.

7 X.-L. Yang, X.-J. Ju, X.-T. Mu, W. Wang, R. Xie, Z. Liu and L.-Y. Chu, ACS Appl. Mater. Interfaces, 2016, 8, 10524-10534.

8 J. M. Weissman, H. B. Sunkara, A. S. Tse and S. A. Asher, Science, 1996, 274, 959-963.

9 W. Qun, F. Shoukuan and Y. Tongyin, Prog. Polym. Sci., 1994, 19, 703-753.

10 J. A. Straub, D. E. Chickering, C. C. Church, B. Shah, T. Hanlon and H. Bernstein, J. Controlled Release, 2005, 108, 21-32.

11 G. M. Whitesides, Nature, 2006, 442, 368-373.

12 T. Y. Lee, T. M. Choi, T. S. Shim, R. A. M. Frijns and S.-H. Kim, Lab Chip, 2016, 16, 3415-3440.

13 L. Y. Chu, A. S. Utada, R. K. Shah, J. W. Kim and D. A. Weitz, Angew. Chem., 2007, 119, 9128-9132.

14 Z. Nie, S. Xu, M. Seo, P. C. Lewis and E. Kumacheva, J. Am. Chem. Soc., 2005, 127, 8058-8063.

15 D. Dendukuri and P. S. Doyle, Adv. Mater., 2009, 21, 4071-4086. 16 S. Takeuchi, P. Garstecki, D. B. Weibel and G. M. Whitesides, Adv. Mater., 2005, 17, 1067-1072.

17 G. Kaufman, R. Boltyanskiy, S. Nejati, A. R. Thiam, M. Loewenberg, E. R. Dufresne and C. O. Osuji, Lab Chip, 2014, 14, 3494-3497.

18 M. Kim, S. J. Yeo, C. B. Highley, J. A. Burdick, P. J. Yoo, J. Doh and D. Lee, ACS Nano, 2015, 9, 8269-8278.

19 Q. Ma, Y. Song, J. W. Kim, H. S. Choi and H. C. Shum, ACS Macro Lett., 2016, 5, 666-670.

20 H. Zhang, E. Tumarkin, R. M. A. Sullan, G. C. Walker and E. Kumacheva, Macromol. Rapid Commun., 2007, 28, 527-538.

21 J. Zhang, R. J. Coulston, S. T. Jones, J. Geng, O. A. Scherman and C. Abell, Science, 2012, 335, 690.

22 E. Kumacheva and P. Garstecki, Microfluidic Reactors for Polymer Particles, John Wiley \& Sons, Ltd, 2011, pp. 109-145, DOI: $10.1002 / 9780470979228 . c h 7$.

23 W. J. Duncanson, T. Lin, A. R. Abate, S. Seiffert, R. K. Shah and D. A. Weitz, Lab Chip, 2012, 12, 2135-2145.

24 T. S. Shim, S.-H. Kim and S.-M. Yang, Part. Part. Syst. Charact., 2013, 30, 9-45.

25 J.-T. Wang, J. Wang and J.-J. Han, Small, 2011, 7, 1728-1754.

26 W. Wang, M.-J. Zhang and L.-Y. Chu, Acc. Chem. Res., 2014, 47, 373-384.

27 G. R. Guillen, Y. Pan, M. Li and E. M. V. Hoek, Ind. Eng. Chem. Res., 2011, 50, 3798-3817.

28 D.-M. Wang and J.-Y. Lai, Curr. Opin. Chem. Eng., 2013, 2, 229-237.

29 P. van de Witte, P. J. Dijkstra, J. W. A. van den Berg and J. Feijen, J. Membr. Sci., 1996, 117, 1-31.

30 B. K. Johnson and R. K. Prud'homme, Aust. J. Chem., 2003, 56, 1021-1024.

31 V. E. Lee, C. Sosa, R. Liu, R. K. Prud'homme and R. D. Priestley, Langmuir, 2017, 33, 3444-3449.
32 C. Zhang, V. J. Pansare, R. K. Prud'homme and R. D. Priestley, Soft Matter, 2012, 8, 86-93.

33 B. Wang, H. C. Shum and D. A. Weitz, ChemPhysChem, 2009, 10, 641-645.

34 J. R. Millman, K. H. Bhatt, B. G. Prevo and O. D. Velev, Nat. Mater., 2004, 4, 98.

35 T. Watanabe, C. G. Lopez, J. F. Douglas, T. Ono and J. T. Cabral, Langmuir, 2014, 30, 2470-2479.

36 C. E. Udoh, V. Garbin and J. T. Cabral, Langmuir, 2016, 32, 8131-8140.

37 E. Chiellini, A. Corti, S. D'Antone and R. Solaro, Prog. Polym. Sci., 2003, 28, 963-1014.

38 E. Prokopová, P. Štern and O. Quadrat, Colloid Polym. Sci., 1985, 263, 899-904.

39 E. Prokopová, P. Štern and O. Quadrat, Colloid Polym. Sci., 1987, 265, 903-907.

40 P. Štern, E. Prokopová and Q. Quadrat, Colloid Polym. Sci., 1987, 265, 234-238.

41 P. Štern, E. Prokopová and O. Quadrat, Colloid Polym. Sci., 1992, 270, 1066-1068.

42 P.-G. d. Gennes, Scaling Concepts in Polymer Physics, Cornell University Press, Ithaca, New York, USA, 1st edn, 1979.

43 D. C. Boris and R. H. Colby, Macromolecules, 1998, 31, 5746-5755.

44 R. H. Colby, Rheol. Acta, 2010, 49, 425-442.

45 C. Harrison, J. T. Cabral, C. M. Stafford, A. Karim and E. J. Amis, J. Micromech. Micromach., 2004, 14, 153.

46 J. T. Cabral, S. D. Hudson, C. Harrison and J. F. Douglas, Langmuir, 2004, 20, 10020-10029.

47 W. Lee, L. M. Walker and S. L. Anna, Phys. Fluids, 2009, 21, 032103.

48 D. L. Bitterfield, A. Utoft and D. Needham, Langmuir, 2016, 32, 12749-12759.

49 R. Stephenson and J. Stuart, J. Chem. Eng. Data, 1986, 31, 56-70.

50 C. E. Udoh, J. T. Cabral and V. Garbin, Sci. Adv., 2017, 3, eaao3353.

51 B. Briscoe, P. Luckham and S. Zhu, Polymer, 2000, 41, 3851-3860.

52 J. N. Mohanty, P. L. Nayak and S. Lenka, Colloid Polym. Sci., 1987, 265, 982-985.

53 A. Salabat and A. Mehrdad, J. Mol. Liq., 2010, 157, 57-60.

54 M. Cable and J. R. Frade, J. Mater. Sci., 1987, 22, 919-924.

55 D. W. Readey and A. R. Copper, Chem. Eng. Sci., 1966, 21, 917-922.

56 P. S. Epstein and M. S. Plesset, J. Chem. Phys., 1950, 18, 1505-1509.

57 A. W. Hixson and J. H. Crowell, Ind. Eng. Chem., 1931, 23, 923-931.

58 P. B. Duncan and D. Needham, Langmuir, 2006, 22, 4190-4197. 59 J. T. Su and D. Needham, Langmuir, 2013, 29, 13339-13345.

60 M. Leeds, Kirk-Othmer Encyclopedia of Chemical Technology, Wiley-Interscience, New York, 2nd edn, 1963.

61 A. Bianco, S. L. Burg, A. J. Parnell, C. M. Fernyhough, A. L. Washington, C. J. Hill, P. J. Smith, D. M. Whittaker, O. O. Mykhaylyk and J. P. A. Fairclough, Langmuir, 2017, 33, 13303-13314. 
62 R. G. Picknett and R. Bexon, J. Colloid Interface Sci., 1977, 61, 336-350.

63 C. Bourges-Monnier and M. E. R. Shanahan, Langmuir, 1995, 11, 2820-2829.

64 L. Pauchard and C. Allain, Europhys. Lett., 2003, 62, 897.

65 L. Pauchard and Y. Couder, Europhys. Lett., 2004, 66, 667.
66 T. Kominami and H. Takata, Dev., Growth Differ., 2004, 46, 309-326.

67 Y. He, S. Battat, J. Fan, A. Abbaspourrad and D. A. Weitz, Chem. Eng. J., 2017, 320, 144-150.

68 A. R. Salmon, R. M. Parker, A. S. Groombridge, A. Maestro, R. J. Coulston, J. Hegemann, J. Kierfeld, O. A. Scherman and C. Abell, Langmuir, 2016, 32, 10987-10994. 